\title{
Vibratory energy exchanges between a system with a chain of Saint-Venant elements and a nonlinear energy sink
}

\author{
C.-H. Lamarque ${ }^{1}$ and A. Ture Savadkoohi ${ }^{1}$ \\ ENTPE, Université de Lyon, LGCB and LTDS UMR CNRS 5513, rue Maurice Audin, F-69518 Vaulx-en-Velin, France
}

\begin{abstract}
We present time multi-scale energy exchanges between a system with chain of Saint-Venant elements and a nonlinear energy sink under periodic external forces. A general analytical methodology is presented to detect the invariant manifold of the system at fast time scale and then its equilibrium and fold singularities at slow time scale. The latter development will let us to predict attraction(s) of the dynamical system at slow time scale to be able to design proper nonlinear energy sink devices for the aim of control and/or energy harvesting.
\end{abstract}

\section{Introduction}

It is proved that by using the nonlinear properties of some light auxiliary oscillators it is possible to trigger vibratory energy and to control main systems under external excitations. One of these nonlinear system (mainely cubic) is names as nonlinear energy sink (NES) and the phenomenon is called energy pumping [1,2]. Non-smooth nonlinear systems due their efficiency and feasibilities in fabrications and design are of highly interests in industrially [3]. Some research works have been carried to show the effectiveness of non-smooth NES devices in control of main structures [4-8]. However, some researches are carried to analyze and to consider the energy pumping phenomenon between a main non-smooth systems and coupled NES (essentially nonlinear or non-smooth) [9-12]. In the current work we try to consider energy pumping phenomenon between a main structure with chain of Saint-Venant elements in parallel and a NES with a a general nonlinearity. Then we narrow our developments to a main system with two Saint-venant elements in parallel which is coupled by a cubic NES. Organization of the paper is as it follow:

\section{The general model of the system}

We consider the system which is depicted in Fig. 1: It consists of two oscillators. The first one with mass, stiffness and damping as $M, k_{0}$ and $\tilde{\lambda}$, respectively which possesses a set of Saint-Venant elements in parallel with characteristics as $\tilde{k}_{j}$ and $\alpha_{j}, j=1,2, \ldots, n$. The second oscillator, namely NES has the mass, stiffness and damping as $m$ $\left(0<\epsilon=\frac{m}{M} \ll 1\right), \tilde{c}_{l}$ and $\tilde{\lambda}_{1}$. The potential of the NES is considered to be nonlinear in general. Governing system of equations of the system reads:

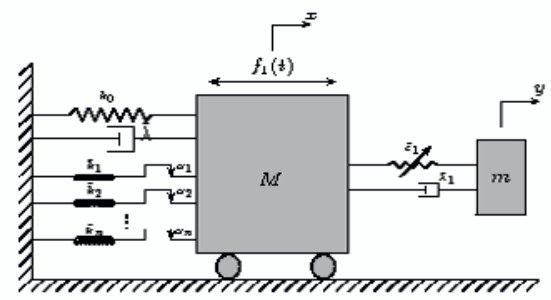

Fig. 1. Two coupled oscillators: the first one with a set of SaintVenant elements and the second one with nonlinear potential.

$$
\left\{\begin{array}{l}
M \frac{d^{2} x}{d t^{2}}+\tilde{\lambda} \frac{d x}{d t}+\tilde{\lambda}_{1}\left(\frac{d x}{d t}-\frac{d y}{d t}\right)+k_{0} x \\
+\sum_{j=1}^{n} \tilde{k}_{j} u_{j}+\tilde{c}_{1} F(x-y)=f_{1}(t) \\
m \frac{d^{2} y}{d t^{2}}+\tilde{\lambda}_{1}\left(\frac{d y}{d t}-\frac{d x}{d t}\right)+\tilde{c}_{1} F(y-x)=0 \\
\left(\frac{d u_{j}}{d t}+\beta\left(\frac{u_{j}}{\eta_{j}}\right)\right) \ni \frac{d x}{d t}, \quad \eta_{j}=\frac{\alpha_{j}}{\tilde{k}_{j}}, \quad j=1,2, \ldots, n
\end{array}\right.
$$

where $F$ is the nonlinear and odd potential of the NES, i.e. $F(-z)=-F(z)$ (e.g. $\left.F(z)=z^{3}\right)$ and the $\beta$ function which is depicted in Fig. 2 can be described as it follows:

$$
\beta(x)= \begin{cases}\emptyset & \text { if } x \in]-\infty,-1[\cup] 1,+\infty[ \\ 0 & \text { if } x \in]-1,1[ \\ \mathbb{R}_{-} \text {if } x=-1 \\ \mathbb{R}_{+} \text {if } x=1\end{cases}
$$

Let us introduce $\tau=t \sqrt{\frac{k}{M}}=w_{1} t, \frac{\tilde{\lambda} w_{1}}{M w_{1}^{2}}=\epsilon \lambda_{0}, \frac{\tilde{k}_{j}}{M w_{1}^{2}}=\epsilon k_{j}$, $\frac{\tilde{c}_{1}}{M w_{1}^{2}}=\epsilon c_{10}, \frac{\tilde{\lambda}_{1}}{M w_{1}^{2}}=\epsilon \lambda_{10}, \frac{f_{1}\left(\frac{\tau}{\omega_{1}}\right)}{M w_{1}^{2}}=\epsilon f_{10} \sin (\Omega \tau)$.

We mention that he differential inclusions of the model un- 
System (6) becomes:

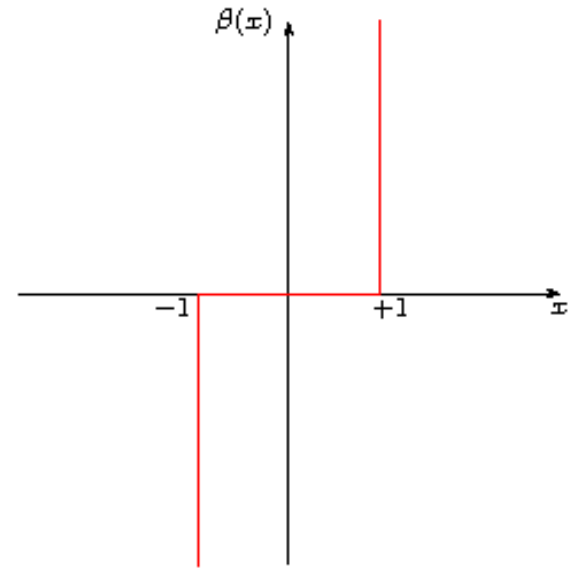

Fig. 2. Graphical representation of the $\beta$ function in Saint-Venant element.

der consideration come from basic constitutive equations of the Saint-Venant elements as:

$$
k_{j} u_{j} \in \alpha_{j} \sigma\left(\frac{d x}{d t}-\frac{d u_{j}}{d t}\right), \quad j=1,2, \ldots, n
$$

where $\sigma$ is the graph of the sign:

$$
\sigma(z)= \begin{cases}-1 & \text { if } z<0 \\ {[-1,1]} & \text { if } z=0 \\ 1 & \text { if } z>0\end{cases}
$$

So one has to take into account that $\frac{d .}{d t}=\omega_{1} \frac{d}{d \tau}$ and

$$
\begin{array}{rr}
\tilde{k}_{j} u_{j} \in \alpha_{j} \sigma\left(\omega_{1}\left(\frac{d x}{d \tau}-\frac{d u_{j}}{d \tau}\right)\right) & j=1,2, \ldots, n \\
\Leftrightarrow \tilde{k}_{j} u_{j} \in \alpha_{j} \sigma\left(\left(\frac{d x}{d \tau}-\frac{d u_{j}}{d \tau}\right)\right) & j=1,2, \ldots, n
\end{array}
$$

Finally Eqs. (1) are equivalent to

$$
\left\{\begin{array}{l}
\frac{d^{2} x}{d \tau_{n}^{2}}+\epsilon \lambda_{0} \frac{d x}{d \tau}+\epsilon \lambda_{10}\left(\frac{d x}{d \tau}-\frac{d y}{d \tau}\right)+x+ \\
\epsilon \sum_{j=1}^{n} k_{j} u_{j}+\epsilon c_{10} F(x-y) \\
=\epsilon f_{10} \sin (\Omega \tau) \\
\epsilon \frac{d^{2} y}{d \tau^{2}}+\epsilon \lambda_{10}\left(\frac{d y}{d \tau}-\frac{d x}{d \tau}\right)+\epsilon c_{10} F(y-x)=0 \\
\left(\frac{d u_{j}}{d \tau}+\beta\left(\frac{u_{j}}{\eta_{j}}\right)\right) \ni \frac{d x}{d \tau}, \quad \eta_{j}=\frac{\alpha_{j}}{\tilde{k}_{j}}, \quad j=1,2, \ldots, n
\end{array}\right.
$$

Let us introduce coordinates of the center of mass and relative displacement via

$$
\left\{\begin{array} { l } 
{ v = x + \epsilon y } \\
{ w = x - y }
\end{array} \Leftrightarrow \left\{\begin{array}{l}
x=\frac{v+\epsilon w}{1+\epsilon} \\
y=\frac{v-w}{1-\epsilon}
\end{array}\right.\right.
$$

$$
\left\{\begin{array}{l}
\frac{d^{2} v}{d \tau^{2}}+\frac{\epsilon \lambda_{0}}{1+\epsilon}\left(\frac{d v}{d \tau}+\epsilon \frac{d w}{d \tau}\right)+\frac{v+\epsilon w}{1+\epsilon}++\epsilon \sum_{j=1}^{n} k_{j} u_{j} \\
=\epsilon f_{10} \sin (\Omega \tau) \\
\frac{d^{2} w}{d \tau^{2}}+\frac{\epsilon \lambda_{0}}{1+\epsilon}\left(\frac{d v}{d \tau}+\epsilon \frac{d w}{d \tau}\right)+\frac{v+\epsilon w}{1+\epsilon}++\epsilon \sum_{j=1}^{n} k_{j} u_{j} \\
+(1+\epsilon)\left(\lambda_{10} \frac{d w}{d \tau}+c_{10} F(w)\right)=\epsilon f_{10} \sin (\Omega \tau) \\
\left(\frac{d u_{j}}{d \tau}+\beta\left(\frac{u_{j}}{\eta_{j}}\right)\right) \ni \frac{1}{1+\epsilon}\left(\frac{d v}{d \tau}+\epsilon \frac{d w}{d \tau}\right), \\
\eta_{j}=\frac{\alpha_{j}}{\tilde{k}_{j}}, \quad j=1,2, \ldots, n
\end{array}\right.
$$

\section{System behavior around 1: 1 resonance}

Let us set $T=\Omega \tau$ and $\cdot=\frac{d}{d \tau}$. We introduce following complex variables [13] to the system:

$$
\begin{array}{ll}
\phi_{1} e^{\imath T}=\Omega(\dot{v}+\imath v) & , \phi_{1}^{*} e^{-\imath T}=\Omega(\dot{v}-\imath v) \\
\phi_{2} e^{\imath T}=\Omega(\dot{w}+\imath w) & , \phi_{2}^{*} e^{-\imath T}=\Omega(\dot{w}-\imath w) \\
\text { for } j=1,2, \ldots, n & \\
\phi_{2+j} e^{\imath T}=\Omega\left(\dot{u}_{j}+\imath u_{j}\right), \phi_{2+j}^{*} e^{-\imath T}=\Omega\left(\dot{u}_{j}-\imath u_{j}\right)
\end{array}
$$

with $t^{2}=-1$. To investigate $1: 1$ resonance, we consider $\Omega=1+\sigma \epsilon$.

We consider only equations obtained by Galerkin method and truncated Fourier series: indeed we take into account only first harmonic $e^{l T}$ for each equation. To calculate corresponding Fourier coefficients we assume that $\phi_{l}$ and $\phi_{l}^{*}$ $(l=1,2, \ldots, n+j)$ do not depend on $T$ : we will either verify this assumption during the multiple scales analysis, or we will assume that after a transient long enough $\phi_{l}$ and $\phi_{l}^{*}(l=1,2, \ldots, n+j)$ reach to an "asymptotic state" independent of $T$. Nevertheless we also keep $\dot{\phi}_{l}$ and $\dot{\phi}_{2}$ in the equations. Then we obtain following system:

$$
\begin{aligned}
& \Omega \dot{\phi_{1}}-\frac{\Omega}{2 \imath} \phi_{1}+\frac{\epsilon \lambda_{0}\left(\phi_{1}+\epsilon \phi_{2}\right)}{2(1+\epsilon)}+\frac{\phi_{1}+\epsilon \phi_{2}}{2 \imath \Omega(1+\epsilon)}+\epsilon \frac{\sum_{j=1}^{n} k_{j} \phi_{j+2}}{2 \Omega l} \\
& =\epsilon \frac{f_{10}}{2 \imath}
\end{aligned}
$$

$$
\begin{aligned}
& \Omega \dot{\phi_{2}}-\frac{\Omega}{2 \imath} \phi_{2}+\frac{\epsilon \lambda_{0}\left(\phi_{1}+\epsilon \phi_{2}\right)}{2(1+\epsilon)}+\frac{\phi_{1}+\epsilon \phi_{2}}{2 \imath \Omega(1+\epsilon)}+\frac{\sum_{j=1}^{n} k_{j} \phi_{j+2}}{2 \Omega l} \\
& +(1+\epsilon)\left(c_{10} \mathcal{F}+\frac{\lambda_{10}}{2} \phi_{2}\right)=\epsilon \frac{f_{10}}{2 l} \\
& \phi_{j+2}=\frac{\phi_{1}+\epsilon \phi_{2}}{(1+\epsilon) \pi} \xi_{j}\left(\frac{\left|\phi_{1}+\epsilon \phi_{2}\right|}{(1+\epsilon) \Omega}\right), \quad j=1,2, \ldots, n
\end{aligned}
$$

where

$$
\mathcal{F}=\frac{1}{2 \pi} \int_{0}^{2 \pi} e^{-l T} F\left(\frac{\phi_{1} e^{\imath T}-\phi_{2}^{*} e^{-l T}}{2 l \Omega}\right) \mathrm{d} T
$$


and $\xi_{j}(z)\left(\forall z \in \mathbb{R}_{+}, j=1,2, \ldots, n\right)$ reads:

$$
\xi_{j}(z)=\left\{\begin{array}{l}
\pi \quad \text { if } z \leqslant \eta_{j} \\
\theta+e^{-l \theta} \sin (\theta)-4 e^{-l \frac{\theta}{2}} \sin \left(\frac{\theta}{2}\right)-\frac{4 \eta_{j}}{z} e^{-l\left(\theta+\frac{\pi}{2}\right)} \\
\quad \text { if } z>\eta_{j}
\end{array}\right.
$$

with

$$
\theta=\arccos \left(1-\frac{2 \eta_{j}}{z}\right)
$$

Now a multiple scale approach [14] with a small (given) parameter $\epsilon$ is presented by considering fast time $T_{0}=T$, and slow times $T_{l}=\epsilon^{l} T, l=1,2, \ldots$ so that

$$
\frac{d}{d T}=\frac{d}{d T_{0}}+\epsilon \frac{d}{d T_{1}}+\epsilon^{2} \frac{d}{d T_{2}}+\ldots
$$

\subsection{Fast time scale: $\epsilon^{0}$-order of the system}

At $\epsilon^{0}$ order, following following equations can be derived from the system of equations (10):

$$
\begin{gathered}
\frac{\partial \phi_{1}}{\partial \tau_{0}}=0 \Rightarrow \phi_{1}=\phi_{1}\left(T_{1}\right) \\
\frac{\partial \phi_{2}}{\partial \tau_{0}}+\frac{\phi_{1}-\phi_{2}}{2 \imath}+c_{10} \mathcal{F}+\frac{\lambda_{10}}{2} \phi_{2}=0 \\
\phi_{j+2}=\frac{\phi_{1}}{\pi} \xi_{j}\left(\left|\phi_{1}\right|\right), \quad j=1,2, \ldots, n
\end{gathered}
$$

We can see from equations that $\phi_{1}$ is a constant versus $T_{0}=$ $T$, as $\phi_{j+2}, j=1,2, \ldots, n$ so that the assumption for the calculation of Fourier coefficients of $e^{l T}=e^{l T_{0}}$ are verified a posteriori. For $\phi_{2}$, we can not claim the same property. This is why we process as it follows: we assume that when $T_{0} \rightarrow \infty, \phi_{2}$ reaches an asymptotic equilibrium governed by a manifold called $T_{0}$ invariant. Then we have:

$$
\frac{\phi_{1}-\phi_{2}}{2 \imath}+c_{10} \mathcal{F}+\frac{\lambda_{10}}{2} \phi_{2}=0
$$

so that implicitly $\phi_{2}$ may depend on $T_{1}$ now, but after long $T_{0}$. So we study modulation of the dynamics around periodic solution depending on time $T_{0}$ associated to the $T_{0}$ invariant. Let us also notice that equations for $\phi_{j+2}, j=$ $1,2, \ldots, n$ are governed by first order differential equations.

\subsection{Slow time scale: $\epsilon^{1}$-order of the system and modulations around $T_{0}$-invariant}

The $\epsilon^{1}$ order of the first equation of system (10) gives:

$$
\frac{d \phi_{1}}{d T_{1}}+\frac{\lambda_{0}}{2} \phi_{1}+\frac{\phi_{2}}{2 \imath}-\frac{2 \sigma+1}{2 \imath} \phi_{1}+\frac{\sum_{j=1}^{n} k_{j} \phi_{j+2}}{2 \imath}=\frac{f_{10}}{2 \imath}
$$

Let us substitute solutions obtained at $\epsilon^{0}$ order for $\phi_{j+2}$, $j=1,2, \ldots, n$ and $T_{0}$-invariant. We write Eq. (18) in the general form:

$$
\phi_{1}=H\left(\phi_{2}, \phi_{2}^{*}\right)
$$

We introduce polar form for $\phi_{j}, j=1,2, \ldots, n+2$ as it follows:

$$
\phi_{j}=N_{j} e^{l \delta_{j}}, N_{j} \in \mathbb{R}_{+}, \delta_{j} \in \mathbb{R}
$$

From relation (20) it is clear that we can obtain two explicit analytical solutions providing $N_{1}$ and $\delta_{1}$ as functions of $N_{2}$ and $\delta_{2}$ :

$$
\begin{aligned}
& N_{1}=H_{1}\left(N_{2}, \delta_{2}\right) \\
& \delta_{1}=H_{2}\left(N_{2}, \delta_{2}\right)
\end{aligned}
$$

From the Eq. (17) we have:

$$
N_{j+2} e^{\imath \delta_{j+2}}=\frac{N_{1}}{\pi} e^{\imath \delta_{1}} \xi_{j}\left(N_{1}\right), \quad j=1,2, \ldots, n
$$

or

$$
N_{j+2} e^{\imath\left(\delta_{j+2}-\delta_{1}\right)}=\frac{N_{1}}{\pi} \xi_{j}\left(N_{1}\right), \quad j=1,2, \ldots, n
$$

so that

$$
N_{j+2}=\frac{N_{1}}{\pi}\left|\xi_{j}\left(N_{1}\right)\right|, \quad j=1,2, \ldots, n
$$

and $\delta_{j+2}$ depends on $N_{1}$ and $\delta_{1}$, let us write

$$
\delta_{j+2}=\rho_{j}\left(N_{1}, \delta_{1}\right), \quad j=1,2, \ldots, n
$$

From (19) we have

$$
\begin{aligned}
& \frac{\partial N_{1}}{\partial T_{1}}+\imath N_{1} \frac{\partial \delta_{1}}{\partial T_{1}}+\left(\frac{\lambda_{0}}{2}-\frac{2 \sigma+1}{2 \imath}\right) N_{1}+\frac{N_{2}}{2 \imath} e^{\imath\left(\delta_{2}-\delta_{1}\right)} \\
& +\frac{\sum_{j=1}^{n} k_{j} \frac{N_{1}}{\pi} \xi_{j}\left(N_{1}\right)}{2 \imath}=\frac{f_{10}}{2 \imath} e^{-\imath \delta_{1}}
\end{aligned}
$$

Introducing real and imaginary parts of $\xi$,

$$
\xi_{j}\left(N_{1}\right)=\xi_{j r}\left(N_{1}\right)+i \xi_{j i}\left(N_{1}\right), \quad j=1,2, \ldots, n
$$

finally one can obtain:

$$
\left\{\begin{array}{l}
\frac{\partial N_{1}}{\partial T_{1}}+\frac{\lambda_{0}}{2} N_{1}+\frac{N_{2}}{2} \sin \left(\delta_{2}-\delta_{1}\right) \\
+\frac{\sum_{j=1}^{n} k_{j} \frac{N_{1}}{\pi} \xi_{j i}\left(N_{1}\right)}{2}=-\frac{f_{10}}{2} \sin \left(\delta_{1}\right) \\
N_{1} \frac{\partial \delta_{1}}{\partial T_{1}}+\frac{2 \sigma+1}{2} N_{1}-\frac{N_{2}}{2} \cos \left(\delta_{2}-\delta_{1}\right) \\
\sum_{j=1}^{n} k_{j} \frac{N_{1}}{\pi} \xi_{j r}\left(N_{1}\right) \\
-\frac{f_{10}}{2} \cos \left(\delta_{1}\right)
\end{array}\right.
$$

Then from (22) we obtain a linear system in $\frac{\partial N_{2}}{\partial T_{1}}$ and $\frac{\partial \delta_{2}}{\partial T_{1}}$ :

$$
\left\{\begin{array}{l}
\frac{\partial H_{1}}{\partial N_{2}} \frac{\partial N_{2}}{\partial T_{1}}+\frac{\partial H_{1}}{\partial \delta_{2}} \frac{\partial \delta_{2}}{\partial T_{1}}-m_{1}=0 \\
H_{1}\left(\frac{\partial H_{2}}{\partial N_{2}} \frac{\partial N_{2}}{\partial T_{1}}+\frac{\partial H_{2}}{\partial \delta_{2}} \frac{\partial \delta_{2}}{\partial T_{1}}\right)-m_{2}=0
\end{array}\right.
$$


where

$$
\begin{aligned}
& -m_{1}=\frac{\lambda_{0}}{2} H_{1}+\frac{N_{2}}{2} \sin \left(\delta_{2}-H_{2}\right)+\frac{\sum_{j=1}^{n} k_{j} \frac{H_{1}}{\pi} \xi_{j i}\left(H_{1}\right)}{2} \\
& +\frac{f_{10}}{2} \sin \left(\delta_{1}\right) \\
& -m_{2}=\frac{2 \sigma+1}{2} H_{1}-\frac{N_{2}}{2} \cos \left(\delta_{2}-H_{2}\right)-\frac{\sum_{j=1}^{n} k_{j} \frac{H_{1}}{\pi} \xi_{j r}\left(H_{1}\right)}{2} \\
& +\frac{f_{10}}{2} \cos \left(\delta_{1}\right)
\end{aligned}
$$

Finally solving equations (29), following system is obtained:

$$
\begin{array}{r}
\frac{\partial N_{2}}{\partial T_{1}}=\frac{\tilde{f}_{1}\left(N_{2}, \delta_{2}\right)}{\tilde{g}\left(N_{2}, \delta_{2}\right)} \\
N_{2} \frac{\partial \delta_{2}}{\partial T_{1}}=\frac{\tilde{f}_{2}\left(N_{2}, \delta_{2}\right)}{\tilde{g}\left(N_{2}, \delta_{2}\right)}
\end{array}
$$

where

$$
\begin{aligned}
& \tilde{f}_{1}\left(N_{2}, \delta_{2}\right)=H_{1} \frac{\partial H_{2}}{\partial \delta_{2}} m_{1}-\frac{\partial H_{1}}{\partial \delta_{2}} m_{2} \\
& \tilde{f}_{2}\left(N_{2}, \delta_{2}\right)=N_{2}\left(\frac{\partial H_{1}}{\partial N_{2}} m_{2}-H_{1} \frac{\partial H_{2}}{\partial N_{2}} m_{1}\right) \\
& \tilde{g}\left(N_{2}, \delta_{2}\right)=H_{1}\left(\frac{\partial H_{1}}{\partial N_{2}} \frac{\partial H_{2}}{\partial \delta_{2}}-\frac{\partial H_{2}}{\partial N_{2}} \frac{\partial H_{1}}{\partial \delta_{2}}\right)
\end{aligned}
$$

The analysis of the dynamical behavior corresponding to a modulation at $1: 1$ resonance around the $T_{0}$-invariant is given by:

- geometry of the $T_{0}$-invariant in the $N_{1}, N_{2}$ and $\delta_{2}$ space associated to the relation $N_{1}=H_{1}\left(N_{2}, \delta_{2}\right)$.

- fixed points of the reduced system (32) are given by:

$$
\left\{\begin{array}{l}
f_{1}\left(N_{2}, \delta_{2}\right)=0, f_{2}\left(N_{2}, \delta_{2}\right)=0 \\
g_{1}\left(N_{2}, \delta_{2}\right) \neq 0, g_{2}\left(N_{2}, \delta_{2}\right) \neq 0
\end{array}\right.
$$

if $f_{1}, f_{2}, g_{1}$ and $g_{2}$ correspond to numerators and denominators of (32).

- singular points of the reduced system (32) are given by:

$$
\left\{\begin{array}{l}
f_{1}\left(N_{2}, \delta_{2}\right)=0, f_{2}\left(N_{2}, \delta_{2}\right)=0 \\
g_{1}\left(N_{2}, \delta_{2}\right)=0, g_{2}\left(N_{2}, \delta_{2}\right)=0
\end{array}\right.
$$

if $f_{1}, f_{2}, g_{1}$ and $g_{2}$ correspond to numerators and denominators of (32). Singular points are potentially associated to bifurcations.

\section{A numerical example}

Let us choose a cubic NES with following characteristics: Let us choose $n=2$ and

$$
F(z)=z^{3}
$$

in such a case, we have

$$
\mathcal{F}=\frac{1}{2 l} G\left(\left|\phi_{2}\right|^{2}\right) \phi_{2}
$$

with

$$
G(x)=\frac{3 c_{10}}{4} x, x \geq 0
$$

and we consider two parallel Saint-Venant elements. It can be proved that is such a case $g_{2} \neq 0$. Let us set $c_{10}=1$, $\lambda_{10}=0.1, \lambda_{0}=0.1, \eta_{1}=0.1, \eta_{2}=0.15, k_{1}=1, k_{2}=2$, $\epsilon=0.001$. We consider external forcing term as $f_{10}=0.7$. Euler's scheme [3,9] with time steps as $\Delta \tau=10^{-4}$ is endowed for solving system of equations (6). Assumed initial conditions are $x(0)=0$ and $y(0)=\dot{x}(0)=\dot{y}(0)=u_{1}(0)=$ $u_{2}(0)=0$. Here present the behavior of the system under higher external forcing terms $f_{10}=0.7$. Predictions of all possible dynamics of the system until reaching to the infinity of the $T_{1}$ time scale are shown in Fig. 3. It is seen that the system has two fold singularities of the first fold line $N_{21}$, namely points 1 and 2 two equilibrium points 3 and 4 and another equilibrium point between two fold lines of the system (unstable area) namely point no. 5. Invariant manifold of the system with corresponding numerical results are presented in Fig. 4. It is seen that the system presents persistence direct and reverse bifurcations between its stability borders. This behavior will be more visible by looking the histories of system amplitudes which are obtained by numerical integration and are illustrated in Fig. 5. This behavior is named as strongly modulated response (SMR) which is due to the existence of fold singularities on the fold line(s) of the system $[7,15]$. Phase portraits of the reduced system (Eq. (32)) around fold singular points no. 1 and 2 are presented in Figs. 6 and 7 shows that these singular points are in the form of saddle and nodes on the fold line of the system $\left(N_{21}\right)$. During SMR both oscillators and all of their components present beating response: displacement histories of two oscillators which are depicted in Fig. 8 and also histories of internal variables of SaintVenant elements which are presented in Fig. 9 show not only beating responses of all components of two oscillators during SMR but also well activations of Saint-Venant elements during energy exchanges between them. The SMR of an optimized designed system is a very desirable behavior from passive control and also energy harvesting view points since both oscillators continue to exchange the energy with large intervals of energy change for the NES and very small energy intervals for the the main system. The system possesses two equilibrium points namely points no. 3 and 4 (see Fig. 3). It can be attracted by one of these points after a very long time at $T_{1}$ time scale or during higher time scales $\left(T_{2}, T_{3}, \ldots\right)$. Due to costly simulation time we did not run it for very long time scales.

\section{Conclusions}

Vibratory energy exchange between two nonlinear systems is studied: The first system contains a chain of parallel Saint-Venant elements. The second oscillator is a nonlinear energy sink with a very small mass with respect to the first oscillator and with a general nonlinear potential. The analysis of the system at fast and also time scales let us obtain its invariant manifold and predict its equilibrium and singular points. These predictions lead us to explain different responses of the system which include bifurcation, attractions to periodic regime and/or strongly modulated responses. The developed technique can be endowed for 


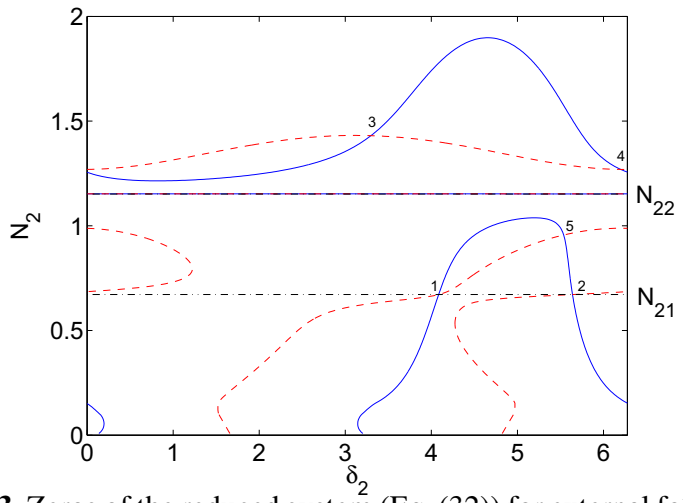

Fig. 3. Zeros of the reduced system (Eq. (32)) for external forcing term $f_{0}=0.7: f_{1}=0(-), f_{2}=0(---), g_{1}=0(-\cdot-\cdot-$, i.e. fold lines $N_{21}$ and $N_{22}$ ).

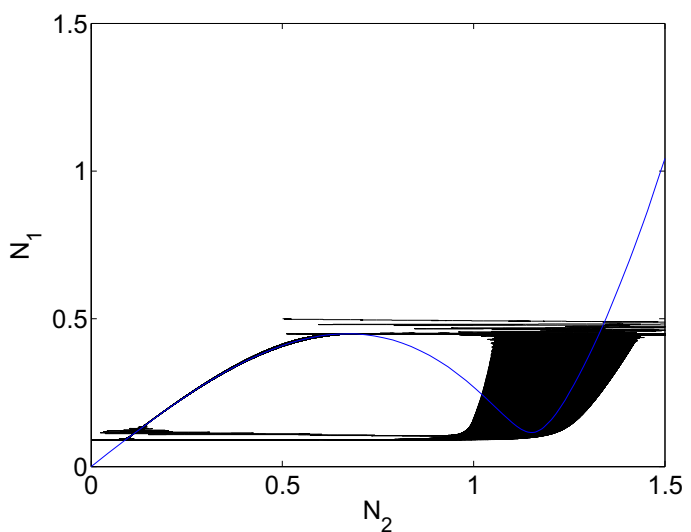

Fig. 4. Analytical invariant manifold of the system (solid blue line) and corresponding numerical results for the system with $f_{0}=0.7$.

designing nonlinear energy sink devices for passively controlling main system which contain friction terms.

\section{References}

1. Vakakis A. F. (2001) Inducing passive nonlinear energy sinks in vibrating systems. ASME Journal of Vibration and Acoustics 123: 324-332.

2. Gendelman O.V., Manevitch L. I., Vakakis A.F., M'Closkey R. (2001) Energy pumping in nonlinear mechanical oscillators I: dynamics of the underlying hamiltonian systems. ASME Journal of Applied Mechanics 68: 34-41.

3. Bastien J., Bernardin F., Lamarque C.-H., Non Smooth Deterministic or Stochastic Discrete Dynamical Systems: Applications to Models with Friction or Impact, 515, Wiley (2013).

4. Nucera F., Vakakis A. F., McFarland D. M., Bergman L. A., Kerschen G. (2007) Targeted energy transfers in vibro-impact oscillators for seismic mitigation. Nonlinear Dynamics 50: 651-677.

5. Lee Y. S., Nucera F., Vakakis A. F., McFarland D. M., Bergman L. A. (2009) Periodic orbits, damped transitions and targeted energy transfers in oscillators with vibro-impact attachments. Physica D 238: 1868-1896.

6. Gendelman O.V. (2008) Targeted energy transfer in systems with non-polynomial nonlinearity. Journal of Sound and Vibration 315: 732-745.

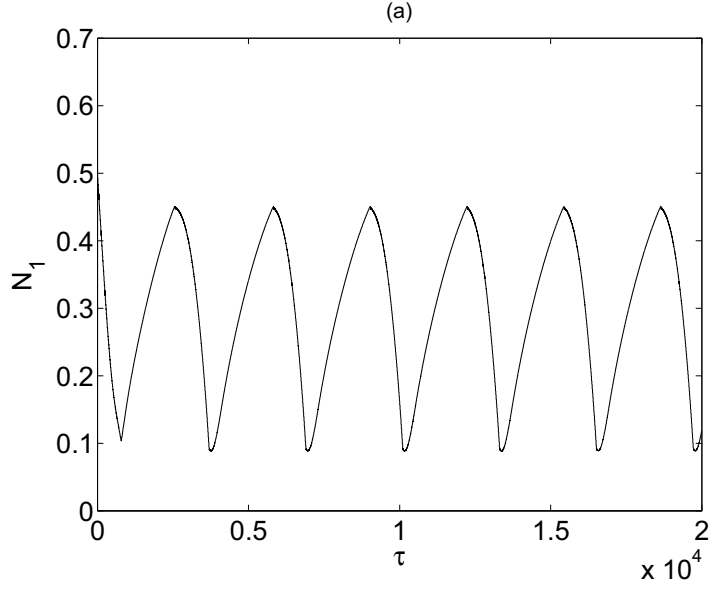

(b)

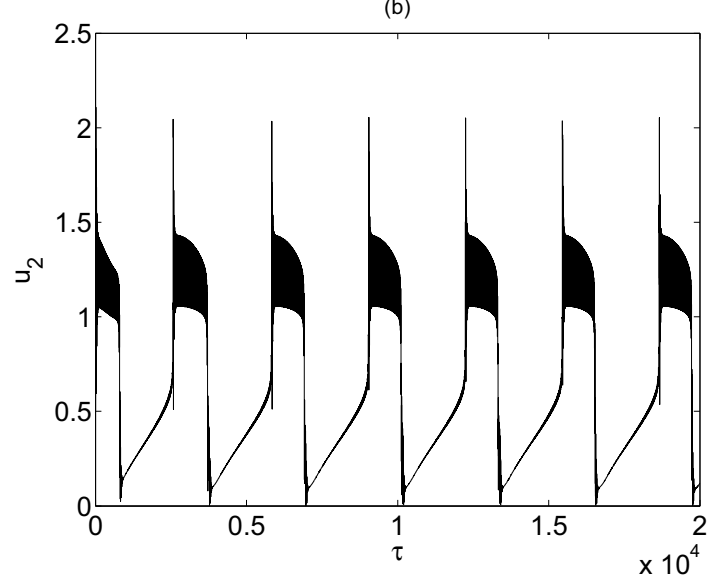

Fig. 5. Numerical values of system amplitudes for the system with $f_{0}=0.7$ : a) $N_{1}$; b) $N_{2}$.

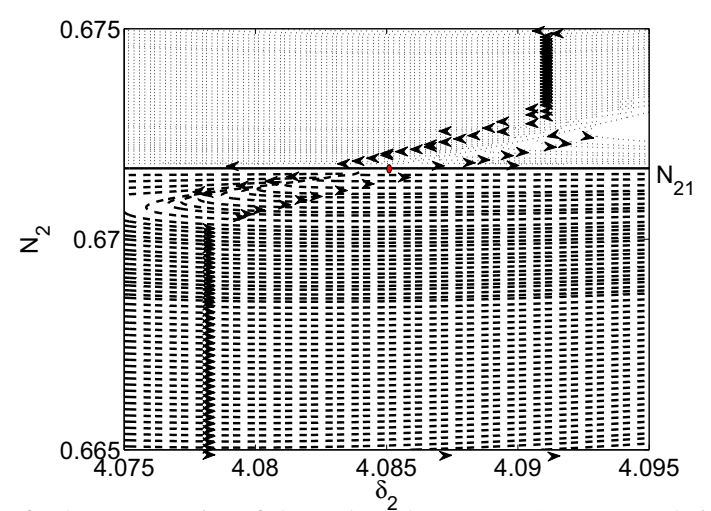

Fig. 6. Phase portraits of the reduced system ((32)) around singular point no. 1 (saddle) with external forcing term $f_{0}=0.7$.

7. Lamarque C.-H., Gendelman O.V., Ture Savadkoohi A., Etcheverria E. (2011) Targeted energy transfer in mechanical systems by means of non-smooth nonlinear energy sink. Acta Mechanica 221: 175-200.

8. Ture Savadkoohi A., Lamarque C.-H., Dimitrijevic Z. (2012) Vibratory energy exchange between a linear and a non-smooth system in the presence of the gravity. Nonlinear Dynamics, 70: 1473-1483.

9. Schmidt F., Lamarque C.-H. (2010) Energy Pumping for Mechanical Systems involving Non-Smooth SaintVenant terms, International Journal of Non-Linear Me- 


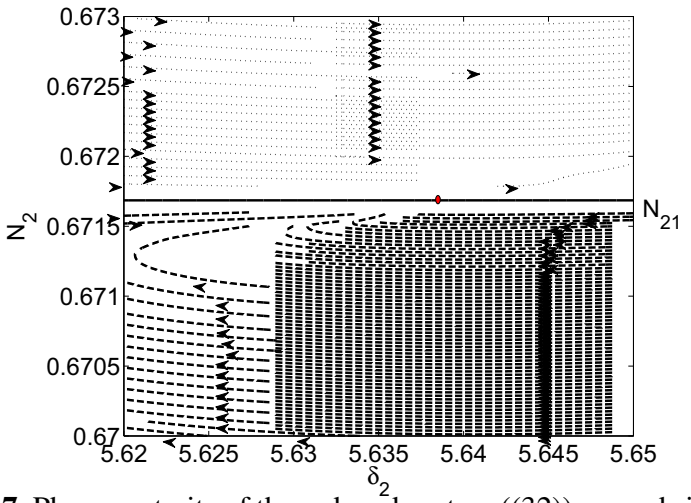

Fig. 7. Phase portraits of the reduced system ((32)) around singular point no. 2 (node) with external forcing term $f_{0}=0.7$.

(a)

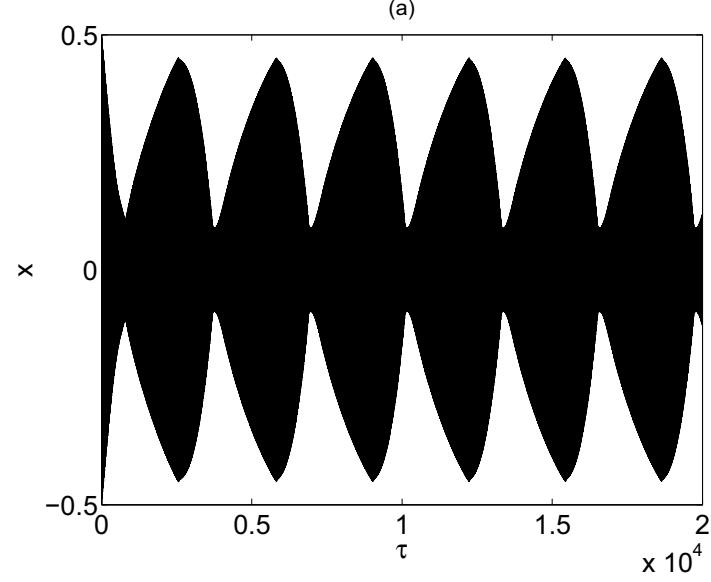

(b)

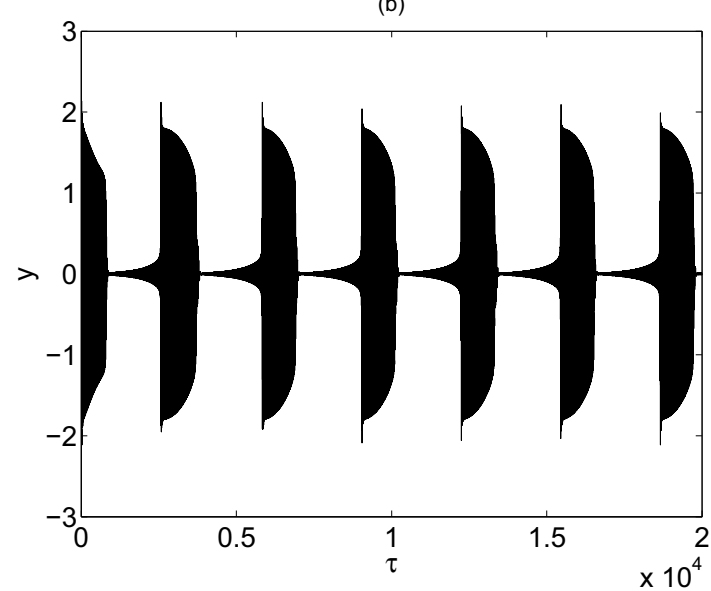

Fig. 8. Numerical values of system displacements for the system with $f_{0}=0.7$ : a) $x$; b) $y$.

chanics, 45: 866-875.

10. Lamarque C.-H., Ture Savadkoohi A., Etcheverria E., Dimitrijevic Z. (2012) Multi-scales dynamics of two coupled non-smooth systems, International Journal of Bifurcation and Chaos, 22: 1250295 (18 pages).

11. Ture Savadkoohi A., Lamarque C.-H. (2013) Dynamics of coupled Dahl type and non-smooth systems at different scales of time, International Journal of Bifurcation and Chaos, 23: 1350114 (14 pages).

12. Lamarque C.-H., Ture Savadkoohi A. (2013) Multiscale energy exchanges between a nonlinear oscillator
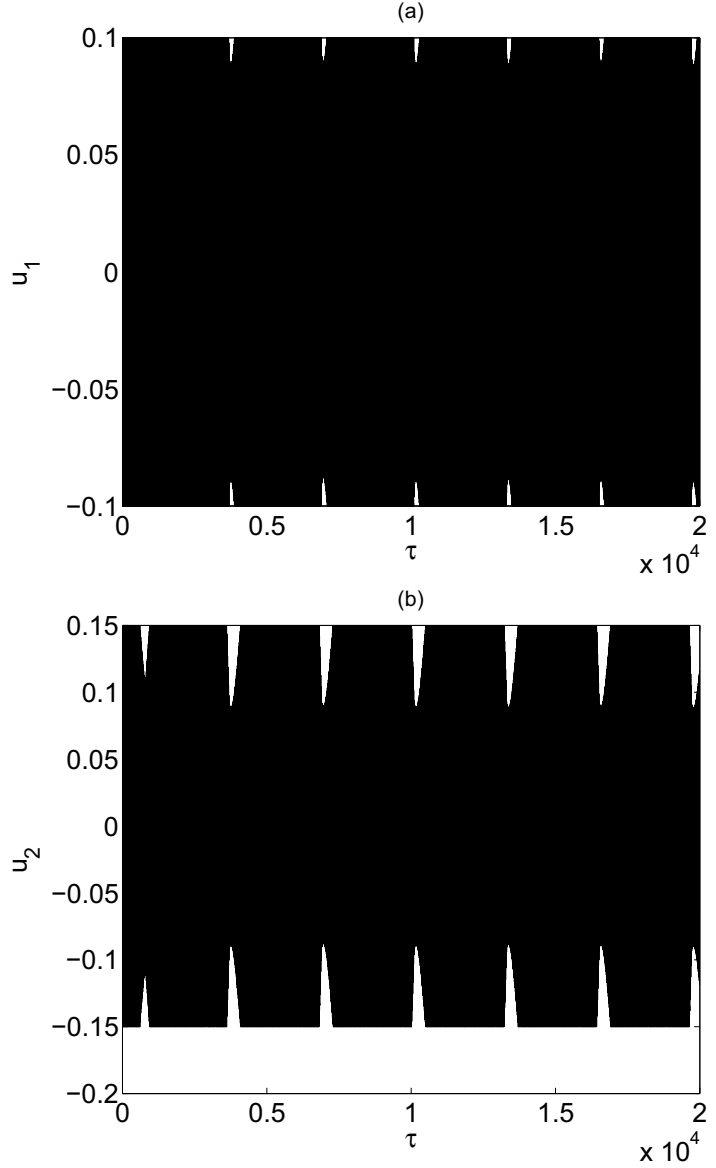

Fig. 9. Numerical values of internal variables of the Saint-Venant element for the system with $f_{0}=0.7$ : a) $u_{1}$; b) $u_{2}$.

of Bouc-Wen type and another coupled nonlinear system, The European Physical Journal Special Topics, 222: 1617-1636.

13. Manevitch L. I. (2001) The description of localized normal modes in a chain of nonlinear coupled oscillators using complex variables. Nonlinear Dynamics 25: 95-109.

14. Nayfeh A. H., Mook D. T. (1979) Nonlinear oscillations. John Wiley and Sons, New York.

15. Starosvetsky Y, Gendelman O. V. (2008) Strongly modulated response in forced 2DOF oscillatory system with essential mass and potential asymmetry. Physica $D$ 237: 1719-1733. 